\title{
Maîtrise des mauvaises herbes de la culture cotonnière par des rotations culturales en Côte d'Ivoire*
}

\author{
Étienne Téhia Kouakou ${ }^{1}$ \\ Dossahoua Traoré2 \\ ${ }^{1}$ CNRA \\ BP 31 \\ Bingerville \\ Côte d'Ivoire \\ <tehiako@yahoo.fr> \\ 2 Université de Cocody-Abidjan \\ 22 BP 582 \\ Abidjan 22 \\ Côte d'Ivoire \\ $<$ traore@ci.reser.org>
}

\begin{abstract}
Résumé
Depuis l'intensification de la culture cotonnière, certaines mauvaises herbes non contrôlées se sont développées abondamment. Un essai de sept rotations culturales a été conduit à la station de recherche du Centre national de recherche agronomique (CNRA) à Bouaké de 2000 à 2002, afin de les maîtriser efficacement. La flore de l'essai a été dominée par Euphorbia heterophylla L. Les rotations maïs-coton-riz et riz-coton-maïs ont permis d'obtenir les niveaux d'enherbement les plus faibles des parcelles. Elles ont fait baisser les densités d'E. heterophylla et de Trianthema portulacastrum L. d'au moins $83 \%$.
\end{abstract}

Mots clés : Côte d'Ivoire ; coton ; lutte culturale ; mauvaise herbe ; rotation culturale.

Thèmes : pathologie ; productions végétales.

\section{Abstract \\ Cotton weed control by crop rotation in Côte d'Ivoire}

Since the intensification of the cotton crop, certain uncontrolled weeds have developed abundantly. A trial with seven crop rotations was carried out at the CNRA cotton research centre in Bouaké, from 2000 to 2002 to find the best weeding methods. Euphorbia heterophylla $\mathrm{L}$. was the main weed of the flora. The best weeding resulted from the following crop rotations: corn-cotton-rice and rice-cotton-corn. They allowed a decrease in population densities of E. heterophylla and Trianthema portulacastrum $L$. of at least $83 \%$.

Key words: cotton; crop rotation; cultural control; Ivory Coast; weeds.

Subjects: pathology; vegetal productions. a culture cotonnière, introduite en Côte d'Ivoire avant 1960, a connu un développement rapide. De 1200 hectares en 1962, les superficies ont atteint 282678 hectares en 2001 avec un rendement moyen de 1,402 t/ha. La modernisation et l'intensification de cette culture ont fait naitre des problèmes d'enherbement qui ont nécessité, dès 1977, la vulgarisation du désherbage chimique. Après plus de vingt années d'utilisation répétée d'herbicides à action principalement graminicide, Euphorbia beterophylla L. et Commelina benghalensis L. sont devenues très envahissants.
Le Nord-Cameroun a connu le même phénomène. Les parcelles de cotonnier de plus de 20 ans, désherbées régulièrement avec l'association dipropétryne + métolachlore à $960+640 \mathrm{~kg} / \mathrm{ha}$ de substance active, ont été totalement dominées par $C$. benghalensis (Le Bourgeois, 1993). Le souci de maitriser les mauvaises herbes de la culture cotonnière a nécessité la recherche d'autres moyens de lutte efficaces. La bonne mầtrise d'E. heterophylla et de C. benghalensis en cultures vivrières avec oxadiargyl à $260 \mathrm{~g} / \mathrm{ha}$ et l'association isoxaflutole + atrazine à 75+1 000 g/ha (Téhia,

\footnotetext{
* Pour citer cet article : Téhia Kouakou E, Traoré D. Maîtrise des mauvaises herbes de la culture cotonnière par des rotations culturales en Côte d'Ivoire. Cah Agric 2010; 19: 200-4. DOI: 10.1684/agr.2010.0397.
} 
communication personnelle) a justifié l'adoption de rotations culturales pour optimiser les moyens de lutte. Ce choix est soutenu par les résultats de Marenco et Santos (1999) qui ont montré que les rotations Crotalaria paulina Schrank-Oryza sativa L. (riz) et Mucuna aterrima Piper et Tracy-O. sativa réduisent l'enherbement, la biomasse sèche des mauvaises herbes et leurs densités de respectivement 70, 80 et $90 \%$, en comparaison avec la culture continue de riz (riz-riz).

Après trois années d'étude de rotations culturales sur la station expérimentale du CNRA (Centre national de recherche agronomique) à Bouaké, leurs effets sur l'enherbement et les densités des mauvaises herbes sont analysés et discutés dans cette publication.

\section{Matériel et méthode}

\section{Matériel végétal}

L'étude a porté sur les mauvaises herbes et sur les cultures utilisées dans les différentes rotations: le cotonnier (Gossypium birsutum, L. Malvaceae), le riz (Oryza sativa, L.. Poaceae), le maïs (Zea mays, L. Poaceae) et l'arachide (Arachis bypogea, L. Fabaceae).

\section{Dispositif expérimental et traitements}

L'essai a été conduit pendant trois ans (2000 à 2002) dans le cadre du programme coton du CNRAà Bouaké ( $7{ }^{\circ} 40$ ' de latitude $\mathrm{N}, 5^{\circ} 00^{\prime}$ de longitude $\mathrm{O}$ ) sur un sol argilosableux avec un pH de 6,6. Un dispositif en blocs de Fisher à quatre répétitions et sept traitements avec des parcelles élémentaires de 76,8 mètres carrés a été adopté. Les traitements étudiés correspondent aux rotations suivantes: maïs-coton-riz (M-C-R), coton-riz-arachide (C-R-A), riz-arachide-maïs (R-A-M), arachidemaïs-coton (A-M-C), riz-coton-maïs (R$\mathrm{C}-\mathrm{M})$, coton-maïs-arachide (C-M-A) et coton-coton-coton (C-C-C).

\section{Conduite des cultures}

Les semis ont eu lieu en juin ou juillet de chaque année. L'oxadiargyl appliqué à $260 \mathrm{~g} / \mathrm{ha}$, la pendiméthaline à $1000 \mathrm{~g} / \mathrm{ha}$, l'association isoxaflutole + atrazine à $75+$ $1000 \mathrm{~g} /$ ha et l'association métolachlore + terbutryne à $1332+668 \mathrm{~g} / \mathrm{ha}$ sont des herbicides de prélevée employés pour désherber respectivement les cultures de riz, d'arachide, de maïs et de cotonnier. Par manque de pendiméthaline, l'arachide a été désherbée en 2000 avec l'association métolachlore + terbutryne à la même dose qu'en culture cotonnière. Les premiers sarclages complémentaires ont eu lieu à 30 jours après semis (JAS).

\section{Notation d'enherbement et relevé floristique}

L'appréciation du niveau d'enherbement des parcelles a été faite chaque année entre 25 et 30 JAS, au moyen de l'échelle CEB (Commission des essais biologiques) modifiée par Marnotte (Marnotte et al., 2004). Les notes, variant de 1 à 9, sont définies ci-dessous en pourcentage de recouvrement du sol : $1: 1$ à $4 \% ; 2: 4,1$ à $11 \% ; 3: 11,1$ à $22,5 \% ; 4: 22,6$ à $40 \%$; 5 : 40,1 à $60 \% ; 6$ : 60,1 à 77,5\%; $7: 77,51$ à $89 \% ; 8: 89,1$ à $96,5 \% ; 9: 96,51$ à $100 \%$. Au cours des notations d'enherbement, des relevés floristiques parcellaires ont été réalisés. Suivant la même échelle, une note d'enherbement a été attribuée à chaque espèce de mauvaise herbe. Les niveaux d'enherbement des parcelles ou des mauvaises herbes ont été caractérisés par les médianes des notes qui représentent, mieux que les moyennes, la valeur centrale d'une distribution (Snedecor et Cochran, 1971).

\section{Mesure de densité}

Pour estimer la densité des mauvaises herbes, des comptages des individus de chaque espèce ont été faits sur chaque parcelle dans quatre placettes de 0,25 mètre carré. Deux placettes sont positionnées dans le deuxième interrang, de part et d'autre de l'interrang central respectivement à trois et neuf pas d'homme du début de la parcelle. Le comptage, méthode utilisable quel que soit le stade de développement des mauvaises herbes (Chicouène, 1999), a été appliqué entre 25 et 30 JAS.

\section{Variation de densité}

Le rapport de la densité parcellaire en 2002 sur la densité initiale témoin en 2000 traduit la variation de densité d'une mauvaise herbe. Il est exprimé en pourcentage. La densité témoin d'une mauvaise herbe est sa densité en 2000 au niveau de la monoculture de cotonnier qui constitue le traitement témoin.

\section{Analyse statistique des données}

Des analyses de variance des densités des mauvaises herbes ont été faites à l'aide du logiciel STAT-ITCF (Institut technique des céréales et fourrages).

\section{Résultats}

Seules les données relatives à $E$. heterophylla, C. benghalensis et Trianthema portulacastrum L. seront présentées, car ce sont les espèces qui ont eu les plus fortes densités. Celles de Cyperus rotundus L. ne seront pas mentionnées dans cet article, car les traitements n'ont eu aucun effet sur elle.

\section{Fréquences et niveaux d'enherbement des mauvaises herbes}

Sur 84 relevés effectués pendant les trois années d'expérimentation, 25 genres et 27 espèces de mauvaises herbes ont été répertoriées. Elles appartiennent à 15 familles dont les plus représentées sont les Asteraceae avec cinq espèces et les Poaceae, Amaranthaceae et Euphorbiaceae avec trois espèces chacune. Les espèces les plus fréquentes sont : $C$. benghalensis (100\%), E. beterophylla (100\%), Ipomoea triloba L. (100\%), Merremia kentrocaulos Rendle (100\%), T. portulacastrum (96\%), C. rotundus (89\%), Desmodium tortuosum (Sw.) DC (82\%), Celosia trigyna L. (61\%), Acanthospermum hispidum de Candolle (61\%) et Brachiaria lata (Schumach.) Hubb. (50\%). Avec une note médiane d'enherbement de 6 en 2000, le niveau d'enherbement d'E. beterophylla a diminué, pour se maintenir à 4 pendant les deux dernières années, toutes parcelles confondues. C. rotundus est la deuxième espèce par l'importance de son niveau d'enherbement annuel qui a progressé de 2 à 3 sur la période 2000 à 2002. Les autres espèces ont eu des niveaux d'enherbement faibles (notes inférieures ou égales à 2). 


\section{Enherbement des parcelles}

Chaque année, de 2000 à 2002, les parcelles cultivées en mais et riz ont connu les plus faibles niveaux d'enherbement: 11 à $60 \%$ (tableau 1), tandis que celles supportant les cultures de cotonnier et d'arachide se sont fortement enherbées : 40 à $96 \%$.

\section{Densités d'E. heterophylla}

En 2000, la densité d'E. heterophylla a été très élevée dans les cultures de cotonnier et d'arachide. Les comptages effectués sur les soles ayant reçu ces deux cultures ont donné $1025,1020,1014$ et 979 individus/mètre carré (tableau 2). Ces valeurs diffèrent hautement de celles obtenues dans les cultures de maïs et de riz, qui se chiffrent respectivement à 240 , 147 et 84 individus/mètre carré.

En 2001, les plus fortes densités d'E. heterophylla ont été trouvées dans la culture cotonnière des parcelles ayant eu les rotations $\mathrm{M}-\mathrm{C}$ et $\mathrm{R}-\mathrm{C}$, dans l'arachide de la rotation R-A et dans la monoculture C-C. Sa densité au niveau de la rotation M-C (260 individus/mètre carré) est significativement plus élevée que celles qui sont atteintes dans les cultures de riz, d'arachide et de maïs des rotations C-R, $\mathrm{R}-\mathrm{A}, \mathrm{A}-\mathrm{M}$ et C-M.

En 2002, c'est dans les cultures d'arachide et de cotonnier des rotations C-R-A, A-M-C et C-M-A qu'on a enregistré encore les plus fortes densités. Les densités atteintes au niveau des rotations A-M-C et

\section{Tableau 1. Notes médianes d'enherbement des parcelles par traitement et par année.}

Table 1. Weed cover median marks in the plots per treatment and per year.

\begin{tabular}{lccccccc}
\hline Années & \multicolumn{7}{c}{ Traitements } \\
\hline & M-C-R & C-R-A & R-A-M & A-M-C & R-C-M & C-M-A & C-C-C \\
2000 & 3 & 8 & 4 & 7 & 4 & 8 & 7 \\
2001 & 6 & 5 & 5 & 4 & 6 & 3 & 6 \\
2002 & 4 & 7 & 5 & 7 & 4 & 8 & 5 \\
\hline
\end{tabular}

$\mathrm{M}$ : maïs ; C : coton ; R : riz ; A : arachide.

Tableau 2. Densités moyennes (nombre de plantes/mètre carré) d'E. heterophylla, suivant les rotations à Bouaké (résultats de 2000, 2001 et 2002).

Table 2. Average densities (plant number $/ \mathrm{m}^{2}$ ) of $E$. heterophylla following crop rotations in Bouaké (results of 2000, 2001 and 2002).

\begin{tabular}{lrrrr}
\hline $\begin{array}{l}\text { Traitements } \\
\text { (Rotations) }\end{array}$ & \multicolumn{3}{c}{$\begin{array}{c}\text { Densité moyenne } \\
\text { (nombre de plantes/m }{ }^{2} \text { ) } \\
\text { d'E. heterophylla par année }\end{array}$} & $\begin{array}{c}\text { Variation } \\
\text { de densité } \\
\text { (\%) }\end{array}$ \\
\cline { 2 - 4 } & $\mathbf{2 0 0 0}$ & $\mathbf{2 0 0 1}$ & $\mathbf{2 0 0 2}$ & \\
\hline M-C-R & $240 \mathrm{~b}$ & $260 \mathrm{a}$ & $11 \mathrm{C}$ & -99 \\
C-R-A & $1025 \mathrm{a}$ & $50 \mathrm{~b}$ & $199 \mathrm{ab}$ & -80 \\
R-A-M & $147 \mathrm{~b}$ & $108 \mathrm{~b}$ & $29 \mathrm{C}$ & -97 \\
A-M-C & $1020 \mathrm{a}$ & $62 \mathrm{~b}$ & $247 \mathrm{a}$ & -75 \\
R-C-M & $84 \mathrm{~b}$ & $155 \mathrm{ab}$ & $17 \mathrm{C}$ & -98 \\
C-M-A & $1014 \mathrm{a}$ & $41 \mathrm{~b}$ & $292 \mathrm{a}$ & -70 \\
C-C-C & $979 \mathrm{a}$ & $186 \mathrm{ab}$ & $78 \mathrm{bc}$ & -92 \\
Moyenne générale & 644 & 123 & 124 & \\
Écart type résiduel & 244 & 65 & 84 & \\
Coefficient de variation en \% & 38 & 53 & 67 & \\
Signification au seuil de 5\% & HS & $\mathrm{S}$ & $\mathrm{HS}$ & \\
\hline
\end{tabular}

$M$ : maïs ; : coton ; R : riz ; A : arachide ; HS: hautement significatif, $S$ : significatif.

NB: Dans les colonnes, les moyennes suivies des mêmes lettres ne diffèrent pas significativement.

C-M-A (247 et 292 individus/mètre carré) diffèrent significativement de celles qui sont obtenues dans les rotations M-C-R, R-A-M, R-C-M et dans la monoculture C-C-C, et qui se chiffrent à 11, 29, 17 et 78 individus/mètre carré. Les plus faibles densités sont obtenues sur les parcelles des rotations M-C-R et R-C-M.

Après trois années d'expérimentation, toutes les rotations étudiées ont permis une réduction de la densité de cette espèce de 70 à 99 \% (tableau 2).

\section{Densités de $\boldsymbol{C}$. benghalensis}

En 2000, les cultures n'ont pas eu d'effet significatif sur les densités de $C$. benghalensis (tableau 3) au seuil $\alpha=5 \%$. En 2001, c'est dans la culture d'arachide de la rotation R-A-M qu'on a compté le plus grand nombre d'individus (16/ mètre carré). Cette densité diffère hautement de celles qui sont obtenues dans les autres rotations. En 2002, cette mauvaise herbe n'a pas été maitrisée dans les cultures d'arachide et de maïs des rotations C-R-A, R-A-M, R-C-M et C-M-A où de nombreuses plantules sont apparues $(21,29$, 17 et 50 individus/mètre carré). À ces valeurs correspondent respectivement des variations de densité de $107 \%$, $192 \%, 67 \%$ et $397 \%$. Les densités obtenues dans les autres rotations ont été plus ou moins faibles (1 à 9 individus/mètre carré). Les densités de $C$. benghalensis ont été très faibles en culture continue de cotonnier et ont varié de 10 individus/mètre carré en 2000 à 1 individu/ mètre carré en 2002, soit une réduction de $90 \%$ (tableau 3).

\section{Densités de $T$. portulacastrum}

En 2000, les densités de T. portulacastrum ont été très élevées dans toutes les soles soumises à la culture cotonnière et dans la monoculture de cotonnier C-C-C. Chiffrées respectivement à 54,55 et 58 individus/mètre carré, elles diffèrent hautement des densités obtenues dans les cultures de maïs et de riz, mais sont comparables à celle qui est trouvée dans la culture d'arachide au seuil de signification de $5 \%$ (tableau 4).

En 2001, c'est dans les cultures de cotonnier et de riz qu'on a trouvé les densités les plus élevées. La densité enregistrée dans le riz (61 individus/mètre carré) a été significati- 
Tableau 3. Densités moyennes (nombre de plantes/mètre carré) de $C$. benghalensis suivant les rotations à Bouaké (résultats de 2000, 2001 et 2002).

Table 3. Average densities (plant number $/ \mathrm{m}^{2}$ ) of $C$. benghalensis following crop rotations in Bouaké (results of 2000, 2001 and 2002).

\begin{tabular}{|c|c|c|c|c|}
\hline \multirow[t]{2}{*}{ Traitements (rotations) } & \multicolumn{3}{|c|}{$\begin{array}{c}\text { Densité moyenne } \\
\text { (nombre de plantes } / \mathrm{m}^{2} \text { ) } \\
\text { de } C \text {. benghalensis, par année }\end{array}$} & \multirow[t]{2}{*}{$\begin{array}{l}\text { Variation } \\
\text { de densité } \\
(\%)\end{array}$} \\
\hline & 2000 & 2001 & 2002 & \\
\hline M-C-R & 27 & $0 \mathrm{~b}$ & $9 \mathrm{~b}$ & -10 \\
\hline C-R-A & 6 & $5 b$ & $21 b$ & 107 \\
\hline R-A-M & 13 & 16 a & $29 a b$ & 192 \\
\hline A-M-C & 14 & $6 \mathrm{~b}$ & $2 \mathrm{~b}$ & -82 \\
\hline R-C-M & 7 & $0 \mathrm{~b}$ & $17 \mathrm{~b}$ & 67 \\
\hline C-M-A & 10 & $4 \mathrm{~b}$ & $50 \mathrm{a}$ & 397 \\
\hline $\mathrm{C}-\mathrm{C}-\mathrm{C}$ & 10 & $0 \mathrm{~b}$ & $1 \mathrm{~b}$ & -90 \\
\hline Moyenne générale & 12 & 4 & 18 & \\
\hline Écart type résiduel & 9 & 4 & 16 & \\
\hline Coefficient de variation en \% & 70 & 91 & 86 & \\
\hline Signification au seuil de $5 \%$ & NS & HS & S & \\
\hline
\end{tabular}

M : maïs; $\mathrm{C}$ : coton; R : riz; A : arachide ; HS : hautement significatif ; $S$ : significatif ; NS : non significatif.

NB: Dans les colonnes, les moyennes suivies des mêmes lettres ne diffèrent pas significativement.

Tableau 4. Densités moyennes (nombre de plantes/mètre carré) de $T$. portulacastrum suivant les rotations à Bouaké (résultats de 2000, 2001 et 2002).

Table 4. Average densities (plant number $/ \mathrm{m}^{2}$ ) of T. portulacastrum following crop rotations in Bouaké (results of 2000, 2001 and 2002).

\begin{tabular}{|c|c|c|c|c|}
\hline \multirow[t]{2}{*}{ Traitements (Rotations) } & \multicolumn{3}{|c|}{$\begin{array}{c}\text { Densité moyenne } \\
\text { (nombre de plantes } / \mathrm{m}^{2} \text { ) } \\
\text { de } T \text {. portulacastrum par année }\end{array}$} & \multirow[t]{2}{*}{$\begin{array}{c}\text { Variation } \\
\text { de densité } \\
(\%)\end{array}$} \\
\hline & 2000 & 2001 & 2002 & \\
\hline $\mathrm{M}-\mathrm{C}-\mathrm{R}$ & $6 \mathrm{~b}$ & $30 a b$ & $2 \mathrm{~b}$ & -97 \\
\hline C-R-A & $54 \mathrm{a}$ & $61 \mathrm{a}$ & $0 \mathrm{~b}$ & -99 \\
\hline R-A-M & $7 \mathrm{~b}$ & $10 \mathrm{~b}$ & $17 \mathrm{ab}$ & -71 \\
\hline A-M-C & $30 a b$ & $2 \mathrm{~b}$ & $1 \mathrm{~b}$ & -98 \\
\hline R-C-M & $3 b$ & $28 a b$ & $10 a b$ & -83 \\
\hline C-M-A & 55 a & $1 \mathrm{~b}$ & $0 \mathrm{~b}$ & -100 \\
\hline $\mathrm{C}-\mathrm{C}-\mathrm{C}$ & 58 a & $43 a b$ & 22 a & -62 \\
\hline Moyenne générale & 30 & 25 & 7 & \\
\hline Écart type résiduel & 18 & 28 & 9 & \\
\hline Coefficient de variation en \% & 59 & 89 & 125 & \\
\hline Signification au seuil de $5 \%$ & HS & S & S & \\
\hline
\end{tabular}

$M$ : maïs ; $C$ : coton ; $R$ : riz ; $A$ : arachide ; HS : hautement significatif ; $S$ : significatif.

NB: Dans les colonnes, les moyennes suivies des mêmes lettres ne diffèrent pas significativement.

vement plus élevée que celles qui ont été obtenues dans l'arachide et le maïs des rotations R-A-M, A-M-C et C-M-A (10, 2 et 1 individus/mètre carré respectivement).
En 2002, les fortes densités ont été enregistrées dans la monoculture de cotonnier et dans le maïs des rotations R-A-M, et R-C-M. La densité obtenue dans la mono- culture de cotonnier est la plus élevée en valeur absolue (22 individus/mètre carré).

Chacune des successions culturales a entraîné chez $T$. portulacastrum une baisse de densité allant de 62 à $100 \%$ (tableau 4).

\section{Discussion}

La bonne maîtrise de l'enherbement des parcelles par les rotations maïs-coton-riz et riz-coton-maïs est due principalement à la très bonne efficacité des herbicides employés dans les cultures de riz et de maîs, notamment l'oxadiargyl appliqué à $260 \mathrm{~g} / \mathrm{ha}$ et l'association isoxaflutole + atrazine appliquée à $75+1000 \mathrm{~g} / \mathrm{ha}$. Koné et al. (1998) et Doucet et al. (1999) ont obtenu des résultats identiques dans des expérimentations similaires.

La forte densité d'E. heterophylla est favorisée par l'importance de son stock semencier et par les conditions permettant la germination et la levée des graines : $\mathrm{pH}$ de 2,5 à 10 , température de 20 à $40{ }^{\circ} \mathrm{C}$, profondeur d'enfouissement jusqu'à 14 centimètres (Brecke, 1995 ; Ipou et al., 2004). Lorsque la température du milieu fluctue, la lumière n'est pas nécessaire à la germination (Brecke, 1995). La terbutryne, qui a remplacé la dipropétryne dans l'association métolachlore + dipropétryne employée au Cameroun (Le Bourgeois, 1993), a permis la réduction de la densité de $C$. benghalensis sur toutes les soles ayant reçu la culture cotonnière.

La densité élevée de T. portulacastrum dans la parcelle de riz en 2001 (61 individus/mètre carré) pourrait s'expliquer par une interaction culture-année, comme l'ont constaté Vecchio et al. (1992) dans une de leurs expérimentations. Cette interaction a probablement été facilitée par la faible pluviométrie de juillet 2001 (31,6 mm), mois où les observations ont été faites. La mauvaise diffusion dans le sol de l'herbicide appliqué le 28 juin 2001 et son exposition au soleil pendant le mois sec de juillet l'ont rendu inefficace à maîtriser T. portulacastrum.

L'inefficacité de l'association métolachlore + terbutryne contre $T$. portulacastrum a favorisé le développement de cette espèce sur les parcelles d'arachide en 2000.

La maitrise des trois mauvaises herbes par les rotations résulte de l'effet combiné des cultures et des modes de désherbage, 
comme le confirme Bachthaler in Beuret (1980) qui signale que l'évolution qualitative et quantitative de la flore adventice des cultures dépend d'un ensemble de facteurs parmi lesquels les techniques culturales sont déterminantes. Les herbicides jouent un rôle primordial dans cette évolution (Doucet et al., 1999). L'efficacité des herbicides permet de comprendre la baisse significative de la densité des mauvaises herbes quand on passe à une culture céréalière après une année de culture de cotonnier ou d'arachide.

La petite saison sèche très marquée de juillet 2001 a contribué à réduire les densités des mauvaises herbes.

Les dates de sarclage influencent aussi l'enherbement et les densités des mauvaises herbes. En effet, les sarclages précoces, réalisés à un moment où les graines des mauvaises herbes ne sont pas encore matures, contribuent à diminuer la quantité de graines pouvant tomber au sol. En conséquence, le stock semencier du sol diminuera également et les levées ultérieures d'adventices seront de moins en moins nombreuses. Dans la présente étude, les sarclages ont eu lieu à $30 \mathrm{JAS}$, pour éviter la maturation des graines d' $E$. heterophylla qui intervient entre 40 et 50 jours après levée (Egunjobi et Kupoluyi, 1973). Ces éléments justifient le délai assez court au bout duquel des changements sont apparus dans la flore de l'essai, contrairement à ce qui a été observé en Europe où les flores adventices de certains essais n'ont commencé à régresser qu'après au moins six années de désherbage chimique (Barralis et Chadoeuf, 1976).

$\mathrm{Au}$ vu des résultats obtenus, pourquoi constate-t-on encore une forte prolifération d'E. heterophylla et de C. benghalensis en milieu paysan où la pratique du désherbage chimique existe depuis plus de 20 ans? L'analyse des pratiques paysannes conduit à mettre en cause le non-respect de la dose de l'herbicide, les conditions de son application et les réalisations tardives des sarclages. Le faible pouvoir d'achat de certains paysans les incite à employer les herbicides à des faibles doses avec des buses coniques sur des terrains souvent mal préparés.

Malgré son efficacité, une monoculture a peu d'avantages par rapport à une rotation culturale qui empêche la sélection d'une flore particulière pouvant devenir très envahissante. La rotation culturale peut aussi, par le choix des cultures, rendre un système de culture performant et durable.

\section{Conclusion}

E. heterophylla, mauvaise herbe très fréquente, a dominé la flore de l'essai. Les rotations maïs-coton-riz et rizcoton-maïs se sont montrées plus efficaces à maîtriser l'enherbement. Ces deux dernières et la rotation riz-arachide-maïs ont assuré un bon contrôle d'E. heterophylla, tandis que $C$. benghalensis n'a été bien maitrisée que par la monoculture de cotonnier. À l'exception de la monoculture de cotonnier qui a été moyennement efficace, toutes les autres rotations étudiées ont permis une forte réduction de la densité de T. portulacastrum.

Cette étude montre qu'un choix bien raisonné de successions culturales comportant à la fois le cotonnier et des cultures nettoyantes (le maïs et le riz) peut aider à résoudre le problème récurrent de l'enherbement des champs de cotonnier. Le succès de ces successions culturales dépend essentiellement de l'efficacité des herbicides employés, de leur parfaite mise en œuvre et de la complémentarité de leurs spectres d'efficacité

La très bonne efficacité d'oxadiargyl sur $E$. heterophylla en culture de riz nécessite de tester sa sélectivité en culture cotonnière.

Si les rotations triennales avec une année de culture cotonnière permettent de mieux maitriser les mauvaises herbes en général, il reste à savoir si ces systèmes de culture sont rentables pour les agriculteurs dont la principale culture commerciale est le cotonnier. Dans le cas contraire, l'adoption d'un assolementrotation biennal, dans lequel le coton serait en rotation avec le maïs ou le riz, peut être envisagée. Avant son adoption, des tests d'arrière-effet des herbicides des cultures céréalières sur le cotonnier devront être réalisés. De plus, cet assolement-rotation doit être étudié sur une longue période - plusieurs rotations complètes - pour connaitre à quel niveau la densité d'adventices se stabilisera.

\section{Références}

Barralis G, Chadoeuf R. Evolution qualitative et quantitative d'un peuplement adventice sous l'effet de dix années de traitement. COLUMA 1976 ; 1 : 179-86.

Beuret E. Influence de la monoculture et des méthodes de travail du sol sur la flore adventice et le stock grainier du sol. COLUMA $1980 ; 2$ : 389-99.

Brecke BJ. Wild poinsettia (Euphorbia heterophylla L.) germination and emergence. Weed Science $1995 ; 43$ : 103-6.

Chicouène $D$. Evaluation du peuplement de mauvaises herbes en végétation dans une parcelle. I-Aperçu des méthodes utilisables. Phytoma 1999 ; 522 : 22-4.

Doucet C, Weaver SE, Hamill AS, Zhang J. Separating the effect of crop rotation from weed management on weed density and diversity. Weed Science $1999 ; 47$ : 729-35.

Egunjobi JK, Kupoluyi AO. Studies on Nigerian weeds. 1. Biology and control of Euphorbia heterophylla L. In: Proceedings of the third Nigeria Weed Science Group Meeting, Lagos, 1973. Samaru : Institute of Agricultural Research (IAR), 1973. www.cabi.org

Ipou IJ, Marnotte P, Aman KG, Aké S, Touré Y. Influence de quelques facteurs environnementaux sur la germination des semences d'Euphorbia heterophylla L. (Euphorbiaceae). Tropicultura $2004 ; 22$ : 176-9.

Koné M, Gakou A, Ballo D, Traoré B. Effets cumulés d'herbicides dans le cadre de rotation culturale au Mali-Sud. COLUMA 1998 ; 2 : 1243-50.

Le Bourgeois TH. Les mauvaises herbes dans la rotation cotonnière au nord-cameroun (Afrique). Amplitude d'habitat et degré d'infestation. Thèse de doctorat de $3^{\mathrm{e}}$ cycle (phénologie), USTL, Montpellier, 1993.

Marenco RA, Santos AMB Crop rotation reduces weed competition and increases chlorophyll concentration and yield of rice. Pesq Agropec Bras 1999; 34 : 1881-7. www.scielo.br/scielo.php

Marnotte P, Perret D, Carrara A. Enquête sur I'enherbement des rizières de Camargue (France). Douzième Colloque international sur la biologie des mauvaises herbes. Dijon : AFPP 2004.

Snedecor GW, Cochran WG. Méthodes statistiques. Paris: Association de coordination technique agricole, 1971.

Vecchio V, Casini P, Calamai P. Dynamique des mauvaises herbes en relation avec la rotation des cultures et les techniques de lutte. Neuvième Colloque International sur la biologie des mauvaises herbes. Dijon : ANPP, 1992. 